HELMINTHOLOGIA, 55, 1: 60 - 69, 2018

\title{
Diplodiscus mehrai Pande, 1937 and D. japonicus (Yamaguti, 1936): morphology of developmental stages and molecular data
}

\author{
V. V. BESPROZVANNYKH' ${ }^{1}$ K. V. ROZHKOVAN ${ }^{1 *}$, A. V. ERMOLENKOํ, A. V. IZRAILSKAYA²
}

${ }^{1}$ Federal Scientific Center of the East Asia Terrestrial Biodiversity FEB RAS, Vladivostok, 690022, Russia, *E-mail: 27.tomcat@gmail.com; '2Department of Biodiversity and marine bioresoures, Far Eastern Federal University, 690051, Vladivostok, Russia

\section{Article info}

Received March 29, 2017 Accepted August 23, 2017

\begin{abstract}
Summary
Specimens of the snails Anisus centrifugops were infected with two types of cercariae relating to the genus Diplodiscus, differing from each other by body and organ sizes were found in a pond in the territory of Vladivostok (Primorsky region, Russia). Further study of their morphology and life cycles allowed us to establish that these flukes belong to Diplodiscus japonicus (Yamaguti, 1936) and $D$. mehrai Pande, 1937. Morphological and molecular data confirmed the validity of $D$. japonicus which had previously been synonymized with D. amphichrus Tubangui, 1933 and D. mehrai Pande, 1937.
\end{abstract}

Keywords: Diplodiscus mehrai; Diplodiscus japonicus; life cycle; cercariae; metacercariae; adult worm

\section{Introduction}

The family Diplodiscidae Cohn, 1904 is a small group of paramphistomoids, found predominantly in amphibians but with representatives in reptiles and in fish (Jones, 2005). The concept of Diplodiscus Diesing, 1836, as well as the taxonomy of individual species of this genus, is based on the morphology of adult worms (Sey, 1991). After revision of Amphistomida, and particularly, Diplodiscus, most of the species and subspecies from this genus described before 1991 were synonymized to different species (Sey, 1991). At present, there is a small amount of data on developmental stages of Diplodiscus. For D. subclavatus (Goeze, 1882), D. amphichrus Tubangui, 1933, D. brevicoeca Richard, Chabaud et Brygoo, 1968 (Sey, 1991) and D. japonicus (Yamaguti, 1936), only cercariae, metacercariae, and parthenitae have been described (Sey, 1991; Skrjabin, 1949). However, the morphometric data for these stages are incomplete. Besides the life cycles of Diplodiscus, data on the circulation features of $D$. subclavatus were obtained. The life span of cercariae after it emergence from a snail (usually in the morning) is approximately $28 \mathrm{~h}$ (Grabda-Kazubska, 1980). Cercariae encyst at the end of the free-living phase. Grabda-Kazubska also showed that cercariae encyst in water, but not on amphibian skin as proposed by Lang (1892), and that the life span of metacercariae is 5 days.

The only species of Diplodiscus known from Russia is D. subclavatus. The literature also reports Diplodiscus sphincterostoma at the territory of the Primorsky Region, discovered by E.V. Belous. However, this fluke is not described; thus, this species was considered a "nomen nudum" by Ryzhikov et al. (1980).

In a pond in the territory of Vladivostok, we found the snail Anisus centrifugops (Prosorova et Starobogatov) with two types of cercariae of Diplodiscus. These cercariae differed in body and organs sizes. Further studies of the life cycles, morphology of different life stages of adult worms allowed us to establish that these flukes belonged to $D$. japonicus and D. mehrai Pande, 1937.

\footnotetext{
$\bar{*}$ - corresponding author
} 


\section{Material and Methods}

\section{Morphology and life cycle}

A total of 250 specimens of Anisus centrifugops naturally infected with parthenitae of species of Diplodiscus were taken from a small pond in the territory of Vladivostok, Primorsky Region, Russia. Adult flukes were also found in naturally infected Rana dybowskii caught in the same place in the spring and autumn. In order to identify these trematodes and their circulation, four infected snails were separately placed in dishes with 1 I of tap water. The snails were divided according to the size of cercariae: two reservoirs contained mollusks with smaller cercariae, and two others contained mollusks with larger ones. Fifteen tadpoles of Rana dybowskii were then placed in each reservoir. Tadpoles were collected in natural ponds free of snails; 30 tadpoles were checked to verify absence of infection before the experiment. During the experiment, tadpoles were periodically dissected to check the level of infection and time of development to the adult worm stage. The process of trematode invasion into the definitive host was studied by placing infected snails into deep Petri dishes. After the peak of cercariae emission, the snail in each dish was replaced with three tadpoles. The process of invasion was observed under a stereo microscope. To check the infection in tadpoles, they were dissected on the first and the subsequent two days from the beginning of the experiment.

To determine the life span of cercariae, infected snails were placed in Petri dishes with tap water for $20-30$ min. Cercariae emerging within this time were counted as even-aged. Maximal cercariae life span was defined by the time of emergence from the mollusk until the death of all specimens. Phototaxy was tested by placing the cercariae into long, narrow glass vials $(20 \times 2 \times 3 \mathrm{~cm}), 1 / 3$ of which were covered by black paper from one side, and a bright spotlight placed on the other side. The diurnal rhythm of cercariae emission was observed by placing a single snail in a Petri dish with $50 \mathrm{ml}$ of tap water. Dishes were shaken every $2 \mathrm{~h}$, and 10 aliquots of $1 \mathrm{ml}$ were taken from each dish and placed in smaller vessels. Dishes were then refilled with water to $50 \mathrm{ml}$. Cercariae were immobilized by iodine and their number in each vessel was counted. The average number of larvae was then multiplied by 5 . Experimental studies were conducted at water temperatures ranging between 18 and $22^{\circ} \mathrm{C}$.

All measurements, in millimeters $(\mathrm{mm})$, of metacercariae and parthenitae were made on living specimens, and before measurements, cercariae were fixed in hot $4 \%$ formalin. Adult worms were fixed in $70 \%$ ethanol and then placed in $96 \%$ ethanol. For whole mounts, worms were stained in alcoholic hydrochloric carmine, dehydrated in a graded ethanol series, cleared in clove oil, and mounted in Canada balsam.

\section{Extraction of total genomic DNA, PCR, and sequencing}

Two and four adult specimens of $D$. mehrai and $D$. japonicus, respectively, were washed several times in a physiological solution immediately after dissection and stored in $96 \%$ ethanol. Genomic DNA was extracted from individual worms using hot sodium hydroxide and Tris (HotSHOT) technique (Truett et al., 2000). The complete sequences of 18S rDNA, ITS1-5.8S-ITS2 rDNA region and partial sequences of $28 \mathrm{~S}$ rDNA were amplified using the following primer sets: 18S-E and 18S-F (Littlewood \& Olson, 2001) for 18S rDNA, BD1 and BD2 (Morgan \& Blair, 1995) for ITS1-5.8SITS2 rDNA, and digl2 and 1500R (Tkach et al., 2003) for $28 \mathrm{~S}$ rDNA. PCR reaction was conducted in a $25 \mu$ l reaction mixture containing $10 \mathrm{pM}$ of each primer, $12.5 \mu \mathrm{l}$ of PCR Master Mix (2X) (Thermo Fisher Scientific Baltics), and $5 \mu$ of extracted DNA. Sequencing was performed on an ABI 3130 automated capillary sequencer (Life Technologies, Grand Island, New York, USA) using PCR primers for $18 \mathrm{~S}$ rDNA and the following internal primers: 373C, 892C, 1262, 1262C, 1/F (Krieger et al., 2006), Pace-A, 18SA27, 18S-4 (Littlewood \& Olson, 2001). ITS1-5.8S-ITS2 rDNA region was sequenced using PCR primers and the internal primer $3 S$ (Littlewood \& Olson, 2001). 28S rDNA was sequenced with ECD2, 900F and 1200R internal primers (Waeschenbach et al., 2007).

\section{Sequence alignment and phylogenetic analyses}

The ribosomal DNA sequences were assembled and aligned manually using the MEGA 5 (Tamura et al., 2011). Sequences have been submitted to GenBank under the following accession numbers: KX506856-KX506857 (D. mehrai) and KX506852-KX506855 (D. japonicus).

For the purpose of phylogenetic analysis we used the combined set of $18 \mathrm{~S}$ and $28 \mathrm{~S}$ rDNA sequences. Prior to phylogenetic analyses sequences were edited, the ends of each sequence were trimmed to match the shortest sequence in the alignment. The final

Table 1. List of taxa, incorporated into the molecular analysis

\begin{tabular}{llll}
\hline Species & $\mathbf{n}$ & Reference & Accession number \\
\hline Diplodiscus mehrai & 2 & Current study & KX506856-KX506857 \\
Diplodiscus japonicus & 4 & Current study & KX506852-KX506855 \\
Diplodiscus subclavatus & 1 & Olson et al., 2003 & AY222212 \\
Fischoederius cobboldi & 3 & Ghatani et al., 2014 & JX518961-JX518963 \\
Fischoederius elongatus & 3 & Ghatani et al., 2014 & JX518964-JX518966 \\
Fasciola hepatica & 1 & Olson et al., 2003 & AY222244 \\
\hline
\end{tabular}


alignment yielded was 2874 nucleotide long and consisted of 13 sequences (Table 1). Sequences of Fasciola hepatica (Linnaeus, 1758) were used as outgroup. Distance matrices were constructed with the absolute pairwise character difference. Pairwise comparisons of absolute sequence divergence for all taxa were calculated with gaps treated as missing data. Maximum Likelihood (ML) using PhyML 3.1 (Guindon \& Gascuel, 2003), and Bayesian Inference (BI) using Mr. Bayes 3.1.2 (Ronquist \& Huelsenbeck, 2003) analyses of the 18S-28S rDNA data were conducted to explore relationships between the newly generated data and nucleotide sequences available in NCBI Genbank. ML and BI phylogenetic analyses were performed using $\mathrm{TIM} 3+\mathrm{G}$ substitution model with gamma correction value 0.1190 . This model showed the best fit to our data using Bayesian information criterion (BIC) in jModeltest 2.1.5 software (Darriba et al., 2012). Bayesian inference was used with the following nucleotide substitution parameters: Iset nucmodel $=4 \mathrm{by} 4$, nst $=6$, and rates = gamma. Markov chain Monte Carlo (MCMC) chains were run for 1,000,000 generations, log-likelihood scores were plotted and only the final $75 \%$ of trees were used to produce the consensus tree. This number of generations was considered sufficient because the standard deviation of spitted sequences value dropped below 0.01 at the end of the run. The significance of the phylogenetic relationship was estimated using a bootstrap analysis (Felsenstein, 1985) with 100 replications for ML

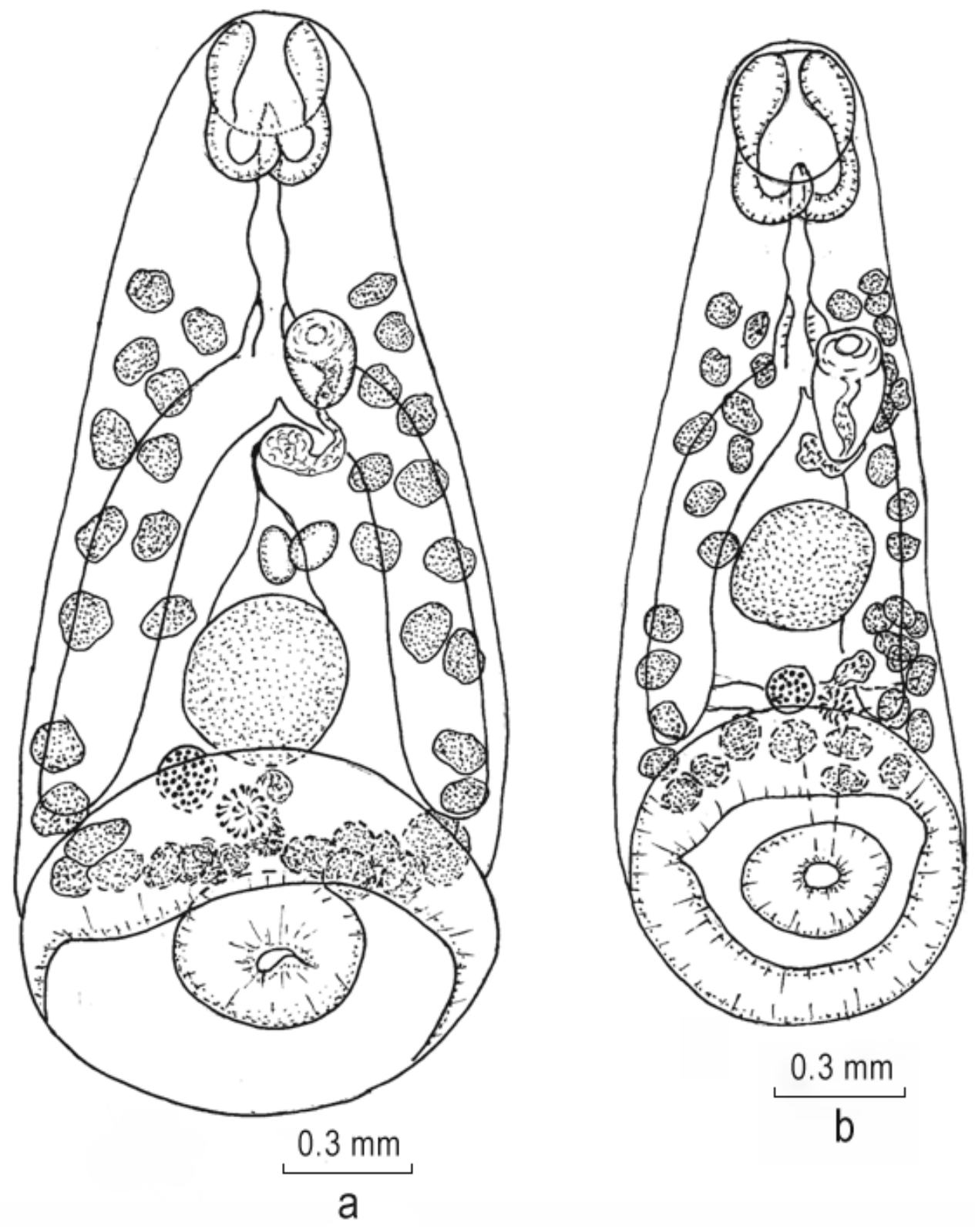

Fig. 1. Adult Diplodiscus: a - D. mehrai Pande, 1937, b- D. japonicus (Yamaguti, 1936). 


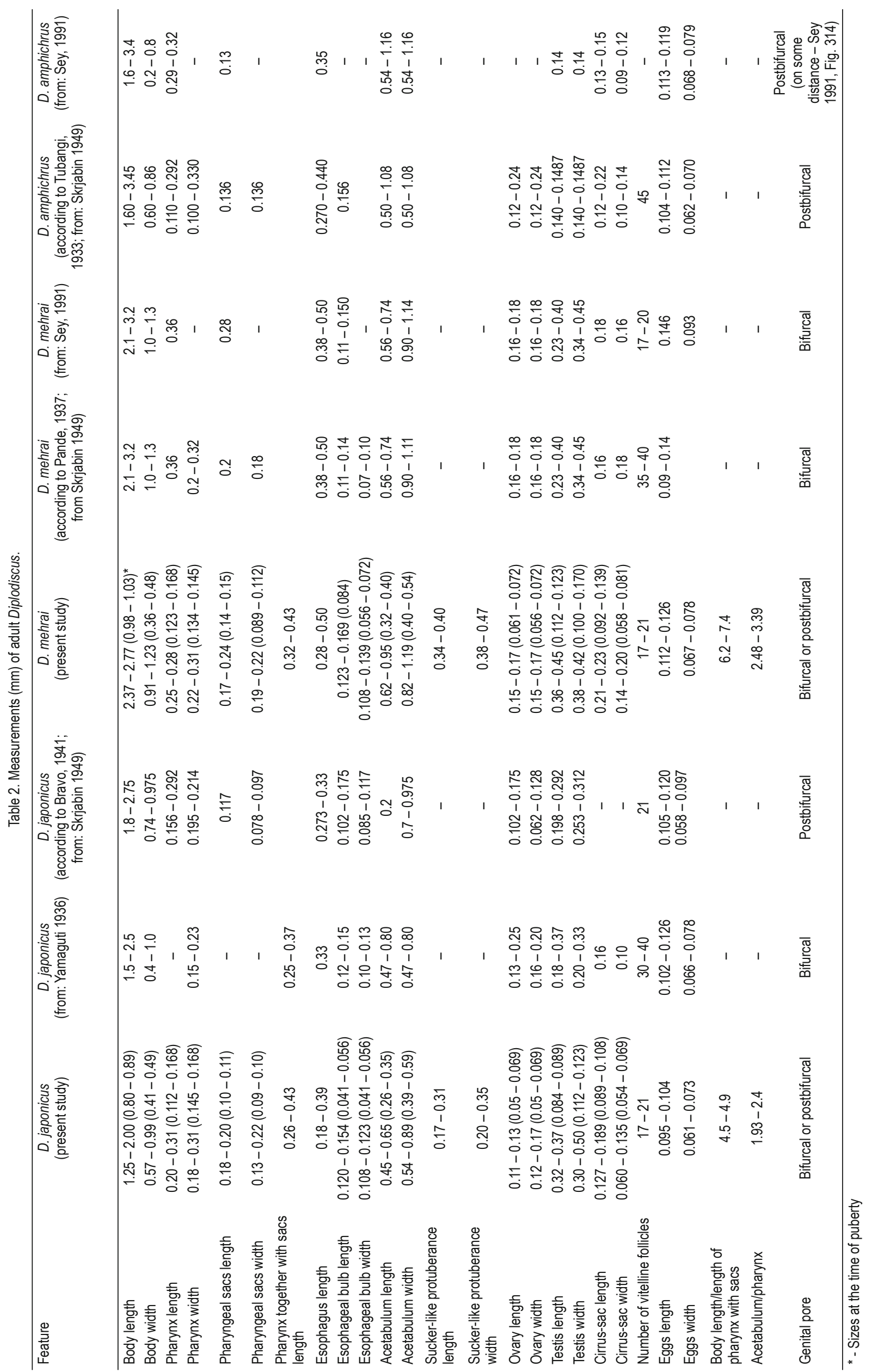


and by the amount of a posterior probability for BI. Trees were visualized using FigTree 1.4 software (http://tree.bio.ed.ac.uk/software/figtree/).

\section{Results}

\section{Description}

\section{Diplodiscus mehrai Pande, 1937}

Definitive host: tadpoles and adult Rana dybowskii Günther (Ranidae, Rafinesque).

Infection rate of tadpoles and frogs in natural conditions: $100 \%$. Infection intensity of tadpoles in natural conditions: $4-23$ specimens.

Infection intensity of frogs in natural conditions: 1 - 15 specimens. Infection rate of tadpoles in experiment: $20 \%$.

Infection intensity of tadpoles in experiment: $1-7$ specimens.

Site: rectum.

Intermediate host: freshwater snail Anisus centrifugops (Prosorova et Starobogatov) (Planorbidae, Rafinesque).

Infection rate: $4.2 \%$.

Locality: Vladivostok, Primorsky Region, Russia; 43¹2’ N, $131^{\circ} 55^{\prime} \mathrm{E}$.

Adult worm (based on 10 specimens; Table 2, Figs. 1a, 3a): Body conical, non-spined with narrow anterior end and wide posterior end. Pharynx terminal with two pharyngeal sacs. Specimens from tadpoles with diffuse pigment spots on both sides of these sacs. Specimens from frogs with pigment spots or not. Oesophagus ventral to pharyngeal sacs. Oesophageal bulb with wall thickness $0.027-0.031$. Oesophageal bifurcation on border of anterior and middle third of body. Caeca reach anterior margin of acetabulum. Acetabulum terminal, on posterior end of body, with central sucker-like protuberance. Testis single, spherical, smooth to irregular, between caeca on median line of body before acetabulum. Cirrus-sac in area of oesophageal bifurcation, with small internal seminal vesicle. External seminal vesicle present, size depends on its occupancy with sexual products. Genital pore bifurcal to postbifurcal, right or left from bifurcation. Ovary dextral from medial line of body on level of posterior margin of testis. Mehlis' gland left from ovary, $0.16 \times 0.15$. Uterus between caeca, genital pore and testis. Each of two vitelline fields form two groups of follicles, one between level of middle of oesophagus and middle of testis, second between posterior margin of testis and acetabulum. Most of second group of follicles in both vitelline fields across body, and follicles reach median line of body. Each vitelline field comprises $17-21$ follicles. Eggs not numerous, operculated. Excretory bladder T-shaped.

Redia (based on 10 specimens; Fig. 2a.) Body sac-shaped, $0.10-$ $0.13 \times 0.28-0.3$. Pharinx $0.09-0.12 \times 0.11-0.12$. Caecum short. Cercaria (based on 10 specimens; Table 3, Figs. 2b, 3b) Body leaf-shaped, slightly transparent, grey by pigment. Pigment eyes

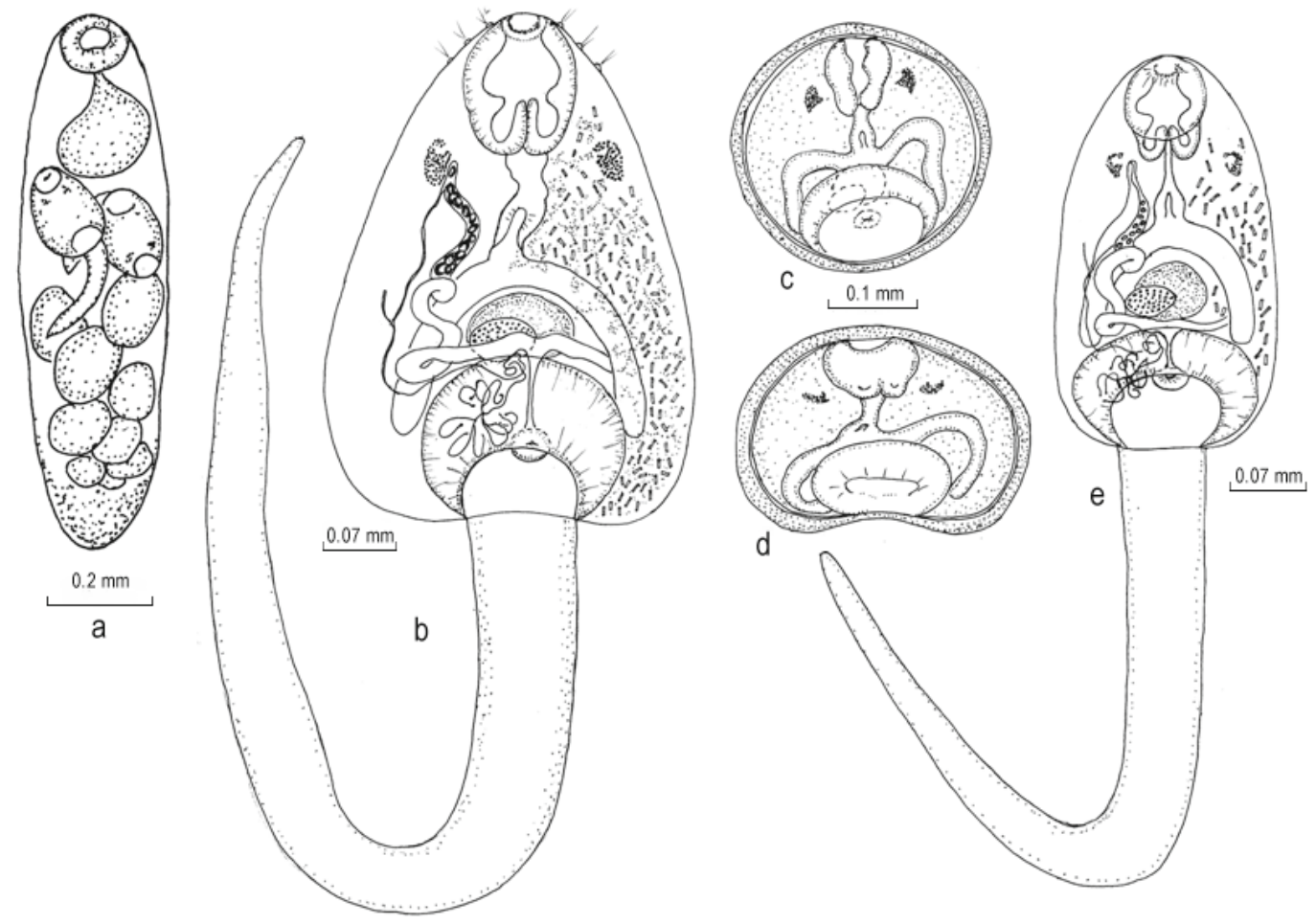

Fig. 2. Diplodiscus mehrai Pande, 1937: a - redia, b - cercaria, c and d-metacercaria, dorso-ventrally and laterally, accordingly; D. japonicus (Yamaguti, 1936): e - cercaria. 


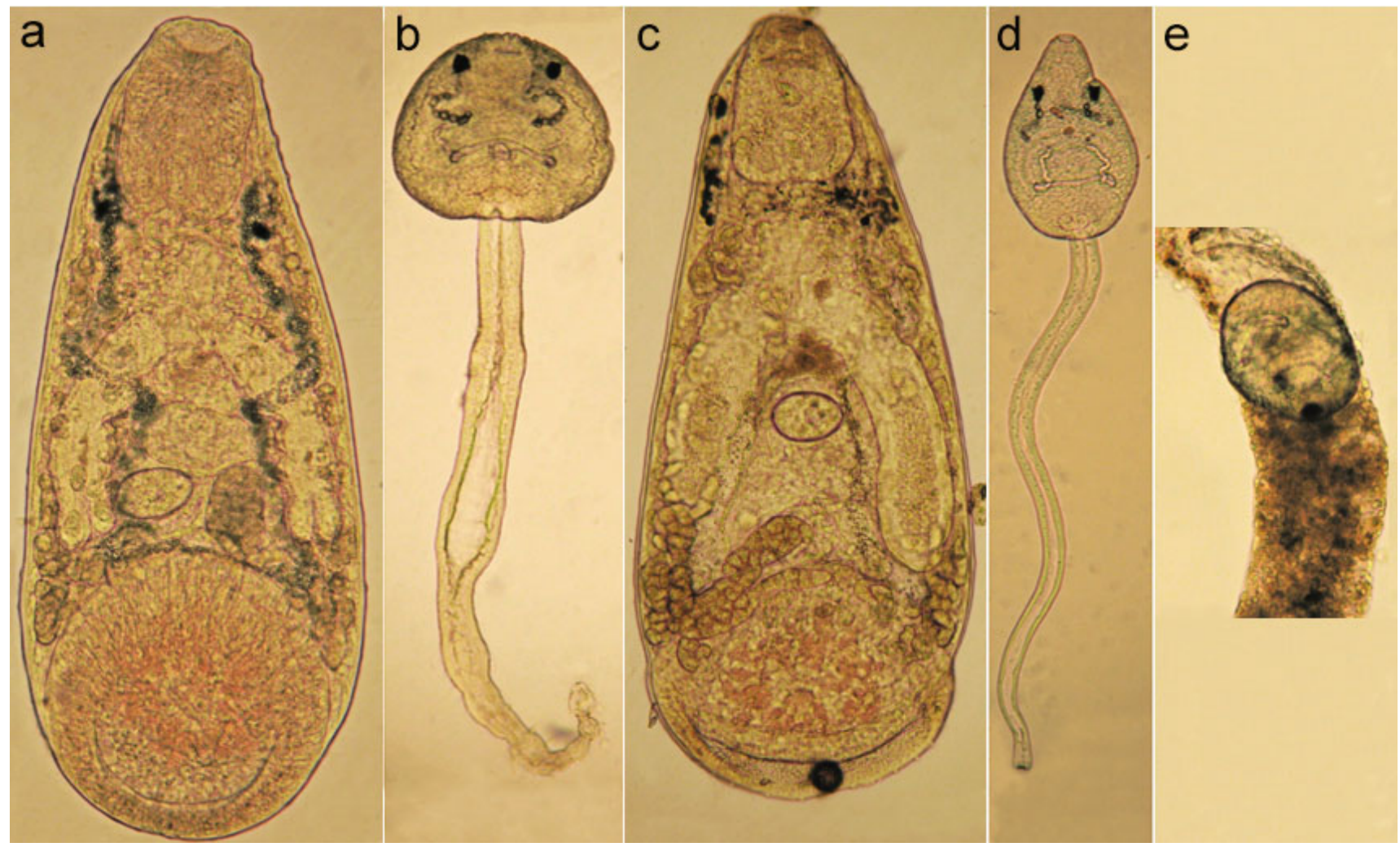

Fig. 3. Diplodiscus mehrai Pande, 1937: a - adult worm, b - cercaria; D. japonicus (Yamaguti, 1936): c - adult worm, d - cercaria; e - metacercaria found in feces of snail.

on both sides of body. Anterior end of body with long sensillae. Pharynx terminal with two sacs. Oesophagus short, with bulb. Oesophageal bifurcation on border of anterior and middle third of body. Caeca terminate at level of middle of acetabulum. Acetabulum terminal, on posterior end of body, with central sucker-like protuberance. Primordium of testis cross-oval, immediately before acetabulum. Primordium of ovary left from median line of body, on level of testis. Cystogenous glands from pharyngeal sacs to posterior end of body. Excretory bladder T-shaped. Two main collective channels, each with $20-21$ granules, left and right from median line of body, turn in area of pigment eyes to posterior end of body. On midline of body each channel divided on two secondary channels; one of them reaches pharynx and second reaches acetabulum, and further divided into three branches, each includes three ducts terminated by small expansion. Tail $1.7-2.1$ times longer than body.

Metacercaria (based on 10 specimens; Fig. 2c,d). Cyst $0.28 \times 0.28$ - 0.29, spherical, concaved on one side.

Molecular sequence data 18S rDNA (complete) - 1991 bp, ITS1 (complete) - 490 bp, 5.8S rDNA (complete) - 159 bp, ITS2 (complete) - 281 bp, $28 \mathrm{~S}$ rDNA (partial) - 1391 bp.

\section{Diplodiscus japonicus (Yamaguti, 1936)}

Definitive host: tadpoles and adult Rana dybowskii Günther (Ranidae, Rafinesque).
Infection rate of tadpoles and frogs in natural conditions: $100 \%$. Infection intensity of tadpoles in natural conditions: 6 - 32 specimens.

Infection intensity of frogs in natural conditions: 1 - 21 specimens. Infection rate of tadpoles in experiment: $43 \%$.

Infection intensity of tadpoles in experiment: 1 - 95 specimens

Site: rectum.

Intermediate host: freshwater snail Anisus centrifugops (Prosorova et Starobogatov) (Planorbidae, Rafinesque).

Rate of infection: $6.4 \%$.

Locality: Vladivostok, Primorsky Region, Russia; $43^{\circ} 12^{\prime} \mathrm{N}, 131^{\circ}$ $55^{\prime} \mathrm{E}$.

Adult worm (based on 10 specimens; Table 2, Figs. 1a, 3c). Body conical or trapezoidal, non spined with narrow anterior end and wide posterior end. Pharynx terminal with two pharyngeal sacs. Specimens from tadpoles with diffuse pigment spots on both sides of these sacs. Specimens from frogs with pigment spots or not. Oesophagus ventral to pharyngeal sacs. Oesophageal bulb oval, with wall thickness $0.039-0.042$. Oesophageal bifurcation on border of anterior and middle third of body. Caeca reach anterior margin of acetabulum. Acetabulum terminal, on posterior end of body, with central sucker-like protuberance, can partly cover ovary. Testis spherical, smooth to irregular, between caeca on median line of body before acetabulum. Cirrus-sac in area of oesophageal 
bifurcation, with small internal seminal vesicle. External seminal vesicle present. Genital pore bifurcal to postbifurcal. Ovary between testis and acetabulum, on median line of body, on some distance from testis. Mehlis' gland left from ovary, $0.11 \times 0.12$. Uterus between caeca, genital pore and ovary. Each of two vitelline fields rorm two groups of follicles. One between level of middle of oesophagus and middle of testis, second between posterior margin of testis and acetabulum Most of second group of follicles in both vitelline fields across body, and follicles reach median line of body. Each vitelline field comprises $17-21$ follicles. Eggs not numerous, operculated. Excretory bladder T-shaped.

Redia (based on 10 specimens). Body sac-shaped, $0.10-0.13 \times$ 0.28 - 0.3. Pharinx $0.09-0.12 \times 0.11-0.12$. Caecum short.

Cercaria (based on 10 specimens; Table 3, Figs. 2e, 3d). Body leaf-shaped, transparent. Pigment eyes on both sides of body. Anterior end of body with poorly visible, short sensillae. Pharynx terminal with two sacs. Oesophagus short, with bulb. Oesophageal bifurcation on border of anterior and middle third of body. Caeca terminate at level of anterior margin of acetabulum. Acetabulum terminal, on posterior end of body, with central sucker-like protuberance. Primordium of testis cross-oval, immediately before acetabulum. Primordium of ovary right from median line of body, on level of testis. Cystogenous glands from pharyngeal sacs to posterior end of body. Excretory bladder T-shaped. Two main collective channels, each with 21 - 23 granules, left and right from median line of body, turn to posterior end of body. On midline of body each channel divided on two secondary channels; one of them reaches pharynx and second reaches acetabulum, and further divided into three branches, each includes three ducts terminated by small expansions. Tail $2.3-2.4$ times longer than body.
Metacercaria (based on 10 specimens). Cyst $0.23-0.24 \times 0.25-$ 0.26 , spherical, concaved on one side.

Molecular sequence data 18S rDNA (complete) - 1991 bp, ITS1 (complete) - 469 bp, 5.8S rDNA (complete) - 159 bp, ITS2 (complete) - 281 bp, $28 \mathrm{~S}$ rDNA (partial) - 1391 bp.

\section{Life cycles}

For both studied species of Diplodiscus, as for other representatives of this genus, the two-host life cycle is usual. Cercariae (1 - 50 specimens) emerged from snails between 11 a.m. and 2 p.m. The most intensive emergences, up to 50 specimens per day, of cercariae occurred during spring. In August - September, this value decreased to 1 - 10 cercariae per day. Larvae are active in water and have positive phototaxis. This allows them to concentrate in warm and sunny pond regions. Such places contain many tadpoles. These factors presumably improve the possibility of definitive host infection. The life span of the free-living form in water temperature $18-22{ }^{\circ} \mathrm{C}$ is $8-12 \mathrm{~h}$. At the end of this time, some cercariae are encysted. The rest, up to $80 \%$, do not encyst and die. Metacercariae did not attach to tadpole skin nor to the bottom of the vessel nor to plants and sand in the vessel, and their life span under laboratory conditions was no longer than 3-4 days. Larvae can frequently be swallowed by snails but are not digested and stay alive. Such metacercariae were found in snail feces (Fig. 3e).

The results of experiments reported here showed that tadpoles infection occurred mainly by swallowing the cercariae. Tadpoles, after being placed with cercariae, swallowed all of them within 20 - $30 \mathrm{~min}$. At this moment, cercariae were not yet encysted and metacercariae were absent. Tadpoles were dissected over 3 days

Table 3. Sizes ( $\mathrm{mm}$ ) of the cercariae and metacercariae of Diplodiscus.

\begin{tabular}{|c|c|c|c|c|c|}
\hline Features & $\begin{array}{l}\text { D. mechrai } \\
\text { (present study) }\end{array}$ & $\begin{array}{l}\text { D. japonicus } \\
\text { (present study) }\end{array}$ & $\begin{array}{c}\text { D. japonicus } \\
\text { (from: Yamaguti 1940) }\end{array}$ & $\begin{array}{l}\text { D. amphichrus (according } \\
\text { to Tubangi \& Masilungan; } \\
\text { from: Sey 1991) }\end{array}$ & $\begin{array}{c}\text { D. amphichrus } \\
\text { (according to Odening; } \\
\text { from: Sey 1991) }\end{array}$ \\
\hline \multicolumn{6}{|c|}{ Cercaria } \\
\hline Body length & $0.51-0.67$ & $0.30-0.36$ & $0.36-0.75$ & $0.21-0.24$ & $0.41-0.59$ \\
\hline Body width & $0.30-0.37$ & $0.19-0.26$ & $0.16-0.35$ & $0.20-0.22$ & $0.27-0.29$ \\
\hline Pharynx with sacs length & $0.12-0.14$ & $0.084-0.127$ & $0.080-0.120$ & - & 0.12 \\
\hline Pharynx width & $0.089-0.095$ & $0.061-0.072$ & $0.054-0.084$ & - & $0.09-0.15$ \\
\hline Pharyngeal bulb length & $0.038-0.045$ & $0.033-0.035$ & $0.027-0.045$ & - & - \\
\hline Pharyngeal bulb width & $0.028-0.034$ & $0.014-0.019$ & $0.020-0.033$ & - & - \\
\hline Acetabulum length & $0.12-0.15$ & $0.112-0.140$ & $0.096-0.165$ & - & $0.15-0.18$ \\
\hline Acetabulum width & $0.22-0.30$ & $0.150-0.178$ & $0.130-0.204$ & - & $0.15-0.18$ \\
\hline Primordium of ovary length & $0.035-0.036$ & $0.028-0.034$ & - & - & - \\
\hline Primordium of ovary width & $0.056-0.058$ & $0.045-0.056$ & - & - & - \\
\hline Primordium of testis length & $0.059-0.062$ & $0.055-0.058$ & 0.030 & - & - \\
\hline Primordium of testis width & $0.089-0.091$ & $0.061-0.063$ & 0.060 & - & - \\
\hline Tail length & $1.12-1.16$ & $0.72-0.83$ & $0.60-0.90$ & $0.76-1.00$ & $0.56-0.69$ \\
\hline Tail widht & $0.11-0.13$ & $0.078-0.089$ & $0.075-0.130$ & - & - \\
\hline \multicolumn{6}{|c|}{ Metacercaria } \\
\hline Cyst & $0.28-0.29$ in diameter & $0.23-0.24 \times 0.25-0.26$ & $0.27 \times 0.25$ & $0.24-0.25 \times 0.20-0.23$ & - \\
\hline
\end{tabular}




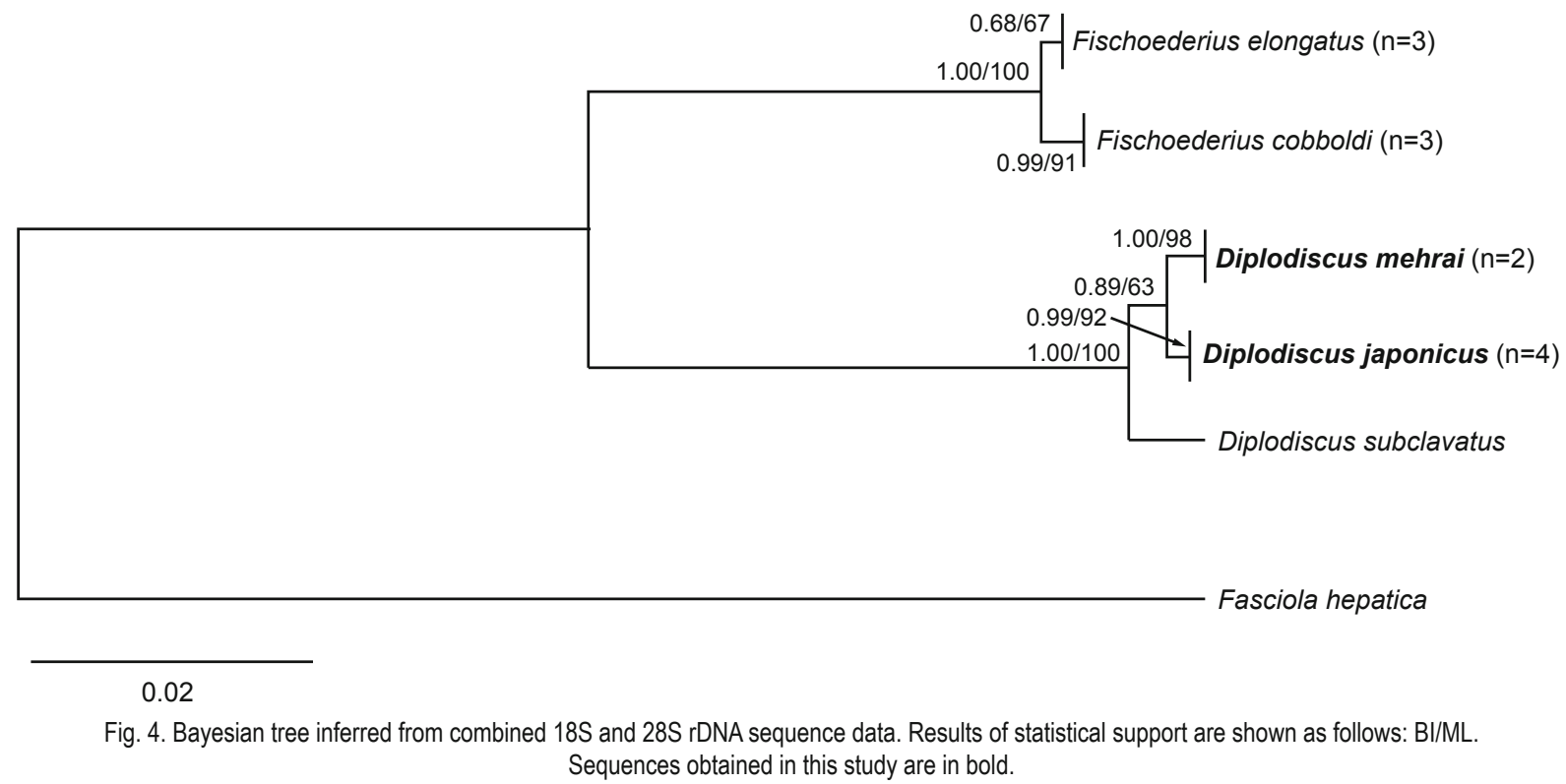

post-exposure, and 7 - 20 flukes were found in their intestines. In other cases, cercariae and metacercariae were found in the tadpoles' oesophagus. Thus, tadpole infection can occur via swallowing either cercariae or metacercariae. However, because only some cercariae are encysted, and the life span of metacercariae is up to 4 days, the primary way of infection occurs through the swallowing of cercariae. Metacercariae can only increase the probability of infection for a short period of time. The first adult $D$. mehrai and $D$. japonicus were both recovered on $30^{\text {th }}$ day of the experiment. Diplodiscus mehrai infected 6 tadpoles with an intensity of 1 to 7 flukes, and $D$. japonicus infected 13 tadpoles with an intensity of 1 to 95 flukes.

According to the natural conditions of the Primorsky Region and biological features of Rana dybowskii, the maximal number of tadpoles in ponds is observed in May - June, and the minimal one is in August - September (Kuzmin, 2012). These periods correspond to maximal and minimal emission of Diplodiscus cercariae, respectively. The tadpoles collected in May - June were infected by both adult flukes and larvae, whereas adult frogs studied in September were infected by adult worms only. This allows us to propose that frog infection in nature occurs at the tadpole stage, and not at adult Rana.

\section{Molecular data}

The 18S-ITS1-5.8S-ITS2-28S rDNA sequence length for $D$. mehrai was $2412 \mathrm{bp}$, and $2391 \mathrm{bp}$ for $D$. japonicus. No intraindividual variation was found in any rDNA region examined. For genetic distances calculation and phylogenetic reconstructions we chose the $18 \mathrm{~S}$ and 28S rDNA genes as the most prevalent in Genbank for the selected group. The main differences between $D$. mehrai and $D$. japonicus were in one $G \leftrightarrow A$ transition for $18 \mathrm{~S}$ rDNA and 8 transitions
(5 $\mathrm{G} \leftrightarrow \mathrm{A}$ and $3 \mathrm{C} \leftrightarrow \mathrm{T}$ ) and one $\mathrm{G} \leftrightarrow \mathrm{T}$ transversion for $28 \mathrm{~S}$ rDNA. The genetic divergence between $D$. mehrai and $D$. japonicus was $0.35 \%$, which is equal to that between Fischoederius cobboldi and $F$. elongatus. The differences relative to other representative of Diplodiscus, D. subclavatus, were 0.87 and $0.8 \%$ for $D$. mehrai and $D$. japonicus, respectively. Phylogenetic analysis (Fig. 4) showed that $D$. mehrai and $D$. japonicus are separate species and they are more closely related to each other than to $D$. subclavatus.

\section{Discussion}

Sey (1991) revised all species belonging to superfamily Paramphistomoidea Fischoeder, 1901 and included 11 species in Diplodiscus. As a result, specimens that had previously been attributed to a single species were synonymized to different species within Diplodiscus (Sey, 1991). In part, this was related to D. mehrai and D. amphichrus japonicus Yamaguti, 1936. From these two species, only D. mehrai, described by Pande in 1937 was included in the 11 valid species within Diplodiscus (Sey, 1991). Specimens found later and identified as $D$. mehrai were synonymized to $D$. magnus (Srivastava, 1934) (Sey, 1991). Li (1937) D. amphichrus japonicus was elevated to species rank and its name was changed to $D$. japonicus. Later, specimens described as $D$. amphichrus japonicus and $D$. japonicus were synonymized either to $D$. amphichrus or D. mehrai (Sey, 1991). All flukes listed above were discovered in East-Asia and India. D. mehrai was also found in Georgia (Petriashvili, 1964).

The results of our research demonstrate the presence of two species of Diplodiscus in the territory of the Primorsky Region. Adult worms of one of these species by metric and morphological characteristics are identical to $D$. mehrai described by Pande (1937) (Table 2). The second species has much in common morphologi- 
cally with $D$. japonicus described by Yamaguti $(1936,1941)$ (Tables 2,3 ). The only significant difference between $D$. japonicus from this study and that of Yamaguti is maximal cercariae body size (Yamaguti, 1940). According to Yamaguti the maximal body size is twice the minimal one. Such differences in size of cercariae are not typical for trematodes from one species. All other morphological traits of cercariae, metacercariae, and adult worms and the participation of snails from Anusis in the circulation of flukes, points to that the second trematode discovered in Primorsky Region, in our opinion, is $D$. japonicus. This species differs from both adult worms and cercariae of $D$. mehrai and $D$. amphichrus by morphometric features. Diplodiscus japonicus differs from adult $D$. mehrai by smaller maximal body size, length of oesophagus, width of ventral sucker and cirrus sac, and maximal egg length. Adult specimens of $D$. japonicus differ from $D$. amphichrus by maximal body size and ventral sucker, testis size, length of pharyngeal sac and position of genital pore (Table 2). Cercariae of $D$. japonicus also have sufficient metric differences from those from $D$. amphichrus and D. ehrai (Table 3).

Thus, in most morphometric characters of the developmental stages, $D$. japonicus differs from both $D$. mehrai and $D$. amphichrus (Tables 2, 3), which allows us to count $D$. japonicus as a separate species within Diplodiscus. The validity of $D$. mehrai and $D$. japonicus is also confirmed by molecular data. Genetic $p$-distances and phylogenetic analysis (Fig. 4) showed that D. mehrai and $D$. japonicus are separate species and that they are more closely related to each other than to $D$. subclavatus.

\section{References}

Darriba, D., Taboada, G.L., Doallo, R., Posada, D. (2012): jModelTest 2: more models, new heuristics and parallel computing. Nat. Methods, 9, 772. DOI:10.1038/nmeth.2109

Felsenstein, J. (1985): Confidence limits on phylogenies: an approach using bootstrap. Evolution, 39, 783 - 791. DOI: $10.2307 / 2408678$

Ghatani, S., Shylla, J.A., Roy, B., Tandon, V. (2014): Multilocus sequence evaluation for differentiating species of the trematode Family Gastrothylacidae, with a note on the utility of mitochondrial COI motifs in species identification. Gene, 548, 277 - 284. DOI: 10.1016/j.gene.2014.07.046

GRABDA-KaZUBSKA, B. (1980): Observation on life-cycle of Diplodiscus subclavatus (Pallas, 1760) (Trematoda, Diplodiscidae). Acta Parasitol., 27, $261-271$

Guindon, S., Gascuel, O. (2003): A simple, fast and accurate algorithm to estimate large phylogenies by maximum likelihood. Syst. Biol., 52, 696 - 704. DOI: 10.1080/10635150390235520

Jones, A. (2005): Family Diplodiscidae Cohn, 1904. In: JonEs, A., Bray, R.A., GIBSON, D.I. (Eds), Keys to the Trematoda. Volume 2. London, UK: CABI Publishing and The Natural History Museum, pp. $319-324$

Krieger, J., Hett, A.K., Fuerst, P.A., Birstein, V.J., Ludwig, A.
(2006): Unusual intraindividual variation of the nuclear 18S rRNA gene is widespread within the Acipenseridae. J. Hered., 97, 218 225. DOI:10.1093/jhered/esj035

KuzMIn, S.L. (2012): [Amphibians of former USSR] Moscow, Fellowship of scientific books. 370 p. (In Russian)

LANG, A. (1892): Über die Cercariae von Amphistomum subclavatum, Ber. Naturforsch. Ges. Freiburg im Breisgau. 9 p.

LI, L.Y. (1937): Some Trematode parasites of frogs with a description of Diplodiscus sinicus sp. nov. Lingnan Sci. Journ. Canton, $19,61-70$

LITTLEWOOd, D.T.J., OLSON, P.D. (2001): Small subunit rDNA and the Platyhelminthes: signal, noise, conflict and compromise. In: LITTLEwOOD, D.T.J. \& BraY, R.A. (Eds), Interrelationships of the Platyhelminthes. London, Taylor \& Francis. pp. $262-278$

MoRgan, J.A.T., BLAIR, D. (1995): Nuclear rDNA ITS sequence variation in the trematode genus Echinostoma: an aid to establishing relationships within the 37-collar-spine group. Parasitology, 111, 609 - 615. DOI: 10.1017/S003118200007709X

Olson, P.D., CRibb, T.H., TKach, V.V., Bray, R.A., LitTlewood, D.T.J. (2003): Phylogeny and classification of the Digenea (Platyhelminthes: Trematoda). Int. J. Parasitol., 33, 733 - 755. DOI: 10.1016/S0020-7519(03)00049-3

PetRIASHVILI, L.G. (1964) [Helminth fauna of the marsh-frog (Rana ridibunda Pall.) in Lake Basaletsky] Soobshch. Akad. Nauk. Gruz. GSSR Vol.36. pp. 457 - 462 (In Russian)

Ronquist, F., HuelsenBeCK, J.P. (2003): MrBAYES 3: Bayesian phylogenetic inference under mixed models. Bioinformatics, 19, 1572 - 1574. DOI: 10.1093/bioinformatics/btg180

RyzhiKov, K.M., Sharpilo, V.P., SheVtschenko, N.N. (1980): [Helminthes of Amphibia of fauna USSR] Moscow, Nauka. pp. 1 - 278 (In Russian)

SEY, O. (1991): CRC Handbook of the zoology of Amphistomes. CRC Press, Inc., Boca Raton, Florida. 480 p.

SkRJABIN, K.I. (1949): Podotriad Paramphistomatata (Szidat, 1936) Skrjabin et Schulz, 1937 [Suborder Paramphistomatata (Szidat, 1936) Skrjabin et Schulz, 1937]. In: SkRJABIN K. I. Trematody zhivotnykh i cheloveka. Osnovy trematodologii [Trematodes of animals and humans]. Moscow, Leningrad, AN SSSR Publ. Volume 3, pp. 1 - 623 (In Russian)

Tamura, K., Peterson, D., Peterson, N., Stecher, G., Nel, M., KuMAR, S. (2011): MEGA5: molecular evolutionary genetics analysis using maximum likelihood, evolutionary distance, and maximum parsimony methods. Mol. Biol. Evol., 28, 2731 - 2739. DOI: 10.1093/molbev/msr121

TKaCh, V.V., LitTlewood, D.T.J., Olson, P.D., Kinsella, J.M., SwidERSKI, Z. (2003): Molecular phylogenetic analysis of the Microphalloidea Ward, 1901 (Trematoda: Digenea). Syst. Parasitol., 56, 1 - 15. DOI: 10.1023/A:1025546001611

Truett, G.E., Heeger, P., Mynatt, R.L., Truett, A.A., Walker, J.A., WARMAn, M.L. (2000): Preparation of PCR-Quality Mouse Genomic DNA with Hot Sodium Hydroxide and Tris (HotSHOT). BioTechniques, $29,52-54$ 
Waeschenbach, A., Webster, B.L., Bray, R.A., Littlewood, D.T.J. (2007): Added resolution among ordinal level relationships of tapeworms (Platyhelminthes: Cestoda) with complete small and large subunit nuclear ribosomal RNA genes. Mol Phyl Evol 45, 311 - 325. DOI: 10.1016/j.ympev.2007.03.019

YamaGutI, S. (1936): Studies on the helminth fauna of Japan. Part 14. Amphibian Trematodes. Jap. J. Zool., 6, 559 - 561

YAMAGUTI, S. (1941): Zur Entwicklungsgeschichte von Diplodiscus amphichrus japonicus Yamaguti, 1936 [History of the development of Diplodiscus amphichrus japonicus Yamaguti, 1936]. Z. Parasitenkd., 11, 652 - 656. DOI: 10.1007/BF02120746 (In German) Zhao, J.H., Wang, S.S., Tu, G.J., Zhou, Y.K., Wu, X.B. (2016): Morphological and molecular characterization of Ortleppascaris sinensis sp. nov. (Nematoda: Ascaridoidea) from the Chinese alligator Alligator sinensis. J. Helminthol., 90,303 - 311. DOI: 10.1017/ S0022149X15000255 